\title{
The adaptation of spike backpropagation delays in cortical neurons
}

\author{
Yossi Buskila ${ }^{1}$, John W. Morley ${ }^{1,2}$, Jonathan Tapson ${ }^{1}$ and André van Schaik ${ }^{1}$ * \\ ' Bioelectronics and Neuroscience Group, The MARCS Institute, University of Western Sydney, Penrith, NSW, Australia \\ ${ }^{2}$ School of Medicine, University of Western Sydney, Penrith, NSW, Australia
}

\section{Edited by:}

Arianna Maffei, State University of New York at Stony Brook, USA

Reviewed by:

Arianna Maffei, State University of New York at Stony Brook, USA Yael Amitai, Ben-Gurion University, Israel

\section{${ }^{*}$ Correspondence:}

André van Schaik, Bioelectronics and Neuroscience Group, The MARCS Institute, University of Western Sydney, Locked Bag 1797, Penrith, NSW 2751, Australia e-mail: a.vanschaik@uws.edu.au
We measured the action potential backpropagation delays in apical dendrites of layer $\checkmark$ pyramidal neurons of the somatosensory cortex under different stimulation regimes that exclude synaptic involvement. These delays showed robust features and did not correlate to either transient change in the stimulus strength or low frequency stimulation of suprathreshold membrane oscillations. However, our results indicate that backpropagation delays correlate with high frequency $(>10 \mathrm{~Hz})$ stimulation of membrane oscillations, and that persistent suprathreshold sinusoidal stimulation injected directly into the soma results in an increase of the backpropagation delay, suggesting an intrinsic adaptation of the backpropagating action potential (bAP), which does not involve any synaptic modifications. Moreover, the calcium chelator BAPTA eliminated the alterations in the backpropagation delays, strengthening the hypothesis that increased calcium concentration in the dendrites modulates dendritic excitability and can impact the backpropagation velocity. These results emphasize the impact of dendritic excitability on bAP velocity along the dendritic tree, which affects the precision of the bAP arrival at the synapse during specific stimulus regimes, and is capable of shifting the extent and polarity of synaptic strength during suprathreshold synaptic processes such as spike time-dependent plasticity.

Keywords: action potential, somatosensory cortex, electrophysiology, cell attached, dendrite, adaptation, rat

\section{INTRODUCTION}

Neurons communicate with each other through the generation of action potentials, which are initiated in the axon initial segment and propagate to the axon terminal (orthodromic propagation), and back into the soma and dendrites (antidromic propagation or backpropagation; Spruston et al., 1995; Stuart et al., 1997b; Bernard and Johnston, 2003). The discovery of a multitude of voltage- and ligand-gated ion channels in the dendrites extended their original concept as passive tubes to include active complex computational properties (Stuart and Sakmann, 1995; Häusser and Mel, 2003; Waters et al., 2005; Froemke et al., 2010), establishing their role as integrative cellular elements. Previous reports showed that the propagation of the antidromic spike along the dendritic arbors depends on the type of neuron, and is highly dependent on $\mathrm{Na}^{+}$conductance along the dendritic tree (Colbert et al., 1997; Stuart et al., 1997a) as well as dendritic morphology (Vetter et al., 2001). Other studies reported on the decremental fashion of the backpropagating action potential (bAP) invasion to the dendrites during a spike train and emphasized the contribution of A-type $\mathrm{K}^{+}$currents in the attenuation of the bAP amplitude along the apical dendrite (Johnston and Hoffman, 1999; Bernard and Johnston, 2003), which could also be modified by the cholinergic agonist charbacol (Tsubokawa and Ross, 1997), suggesting network driven neuromodulation of the antidromic propagation. On the other hand, a recent in vivo study showed that bAP invasion to the distal dendrites is highly reliable across a wide range of brain states, network activity and stimulus conditions and is only mildly modulated by neuronal firing frequency, hence it is well suited to provide crucial signals for the control of synaptic plasticity (Bereshpolova et al., 2007).

In recent years, several studies have shown that the antidromic propagation of action potentials into the dendrites could significantly impact the way signals are processed in the central nervous system (Häusser and Mel, 2003; Nevian et al., 2007; Sjöström et al., 2008). More specifically it has been shown that spike backpropagation provides an associative link between the axonal output and the synapses, thus allowing them to follow a "Hebbian learning rule" such as spike time-dependent plasticity (STDP; Magee and Johnston, 1997; Dan and Poo, 2004; Nevian et al., 2007). In that regard, bAP in the dendrites has been demonstrated to be an important postsynaptic signal for the generation of long-term potentiation (LTP) in several brain regions such as hippocampus (Magee and Johnston, 1997) and neocortex (Markram et al., 1997; Stuart and Häusser, 2001; Sjöström et al., 2008). During backpropagation, the spikes cause depolarization along the dendrite and can interact with orthodromic signals arriving from the synapse to initiate calcium electrogenesis (Larkum, 1999). Therefore, the precise time of arrival of the bAP at the location of the synaptic input will determine the extent and polarity of any change in synaptic strength. Hence, the precision of the backpropagating spike is most important during situations in which the time window between the pre and postsynaptic signals is very short. In these cases, even slight alterations in the backpropagation delay, which are due to modification of the conduction velocity along the apical dendrite, will impact the delay time of the peak amplitude, which will result in modifications of the associated calcium electrogenesis and signal 
integration at the synapse, thus shifting the synaptic gain (Dan and Poo, 2006).

Since Hebbian synaptic plasticity acts as a positive feedback mechanism, it leads to destabilization of the neuronal network (Narayanan and Johnston, 2010). A recent simulation study argues that as a compensation for this instability, there is a modification of the synaptic threshold in an activity-dependent manner (Narayanan and Johnston, 2010), as proposed in the BienenstockCooper-Munro (BCM)-like plasticity framework (Bienenstock et al., 1982), which is achieved through regulation of the $I_{\mathrm{h}}$ current in the dendrites. In this way, the threshold modulation acts as a negative feedback and returns the stability to the network. As an outcome of this regulation, it is argued that the membrane excitability will be modified. If so, this would affect the bAP propagation delay.

As the bAP is context specific (i.e., depends on the state of $\mathrm{Na}^{+}$and $\mathrm{K}^{+}$channels and the recent history of the membrane potential; see Bernard and Johnston, 2003), and may carry contextual information, we studied the adaptation of spike backpropagation delays to various stimuli, including suprathreshold stimulus currents that mimicked the pairing between the excitatory postsynaptic potential (EPSP) and somatic spike as occurs in the soma during STDP inducing protocols (Dan and Poo, 2004). The bAP delays correlate linearly with high frequency $(>10 \mathrm{~Hz})$ stimulation of membrane oscillations as seen in vivo (Bereshpolova et al., 2007). Although transient changes in the suprathreshold sinusoidal stimulus (SSS) frequency $(<10 \mathrm{~Hz}) \mathrm{did}$ not correlate with bAP delays, persistent injection of low frequency (7 Hz) suprathreshold sinusoidal current into the soma result in significant alterations of the bAP delay, suggesting an intrinsic adaptation of the bAP, which does not involve any synaptic modifications.

Some of the results have been published previously in abstract form (Buskila et al., 2013).

\section{MATERIALS AND METHODS ANIMALS}

For this study, we used 2-5 weeks old Wister rats. All animals were healthy and handled with standard conditions of temperature, humidity, $12 \mathrm{~h}$ light/dark cycle, free access to food and water, and without any intended stress stimulations. All experiments were approved by the University of Western Sydney committee for animal use and care [Animal Care and Ethics Committee (ACEC) protocol \#A9452].

\section{SLICE PREPARATION AND RECORDING}

Wister rats were deeply anesthetized by inhalation of isoflurane (5\%), decapitated, and their brains were quickly removed into icecold physiological solution (artificial cerebrospinal fluid, aCSF) containing (in mM): $125 \mathrm{NaCl}, 2.5 \mathrm{KCl}, 1 \mathrm{MgCl}_{2}, 1.25 \mathrm{NaH}_{2} \mathrm{PO}_{4}$, $2 \mathrm{CaCl}_{2}, 25 \mathrm{NaHCO}_{3}, 25$ dextrose and saturated with carbogen (95\% $\mathrm{O}_{2}-5 \% \mathrm{CO}_{2}$ mixture; $\mathrm{pH}$ 7.4). Parasagittal brain slices (300 $\mu \mathrm{m}$ thick) encompassing the primary somatosensory cortex were cut with a vibrating microtome (Camden Instruments, UK) and transferred to a holding chamber containing carbogenated aCSF for $30 \mathrm{~min}$ at $35^{\circ} \mathrm{C}$, which was then allowed to cool to room temperature for at least $1 \mathrm{~h}$ before recording.

\section{ELECTROPHYSIOLOGICAL RECORDINGS AND STIMULATION}

The recording chamber was mounted on an Olympus BX-51 microscope equipped with IR/DIC (infrared/differential interference contrast) optics. During recordings, the slices were kept at room temperature, $\sim 22^{\circ} \mathrm{C}$, and constantly perfused $(2-3 \mathrm{ml} / \mathrm{min}$ ) with oxygenated solution as reported previously (Buskila and Amitai, 2010). Simultaneous whole-cell and cell-attached recordings were made from the soma and dendrites of neurons, respectively. Whole-cell recordings were performed from the soma of layer $\mathrm{V}$ pyramidal neurons in the somatosensory cortex with patch pipettes (5-7 M $\Omega$ ) containing (in $\mathrm{mM}$ ) $130 \mathrm{~K}$-methansulfate, 10 HEPES, 0.05 EGTA, $7 \mathrm{KCl}, 0.5 \mathrm{Na}_{2}$ GTP, $2 \mathrm{Na}_{2}$ ATP, 2 MgATP, 7 phosphocreatine, 0.1 Alexa Fluor-488 (Molecular Probes) and titrated with $\mathrm{KOH}$ to $\mathrm{pH} 7.2$ ( $\sim 285 \mathrm{mOsm})$. Cell-attached recordings from the apical dendrites were performed using patch pipettes (10-12 $\mathrm{M} \Omega$ ) filled with the same internal solution, excluding the fluorescent dye. The use of cell-attached patch recordings rules out the possibility that the findings are caused by washout of cytoplasmic constituents or capacitive load imposed on the cell by the patch pipettes. Stimulation protocols were designed using pClamp 10 software suit (Molecular Devices, Sunnyvale, CA, USA) and stimulation currents were injected through the recording electrodes. Voltages were recorded in current clamp mode using a multiclamp 700B dual patch-clamp amplifier (Axon Instruments, Foster City, CA, USA), digitally sampled at 30$50 \mathrm{kHz}$, filtered at $10 \mathrm{kHz}$, and analyzed off-line using pClamp software. The access resistance was corrected on-line and recordings were included in the analysis if the access resistance was $<30 \mathrm{M} \Omega$, and were considered stable and suitable for analysis if the access resistance, input resistance, and resting membrane potential did not change by more than $20 \%$ from their initial value during recording. At the termination of each experiment, the location and morphology of neurons were examined by fluorescence microscopy and digitally recorded (ROLERA-XR, Q-Imaging).

\section{DETERMINING THE RESONANCE FREQUENCY}

In order to reveal the resonance frequency of the cells, we used the impedance analysis described by Gutfreund et al. (1995). In short, a 20-s subthreshold sinusoidal current with a linear increase in frequency from 0.1 to $20 \mathrm{~Hz}$ (chirp stimulation) was applied through the recording electrode. The impedance amplitude profile (ZAP) was generated by transforming the input current $(I)$ and the voltage response $(V)$ into the frequency domain using a fast Fourier transform (FFT), and then dividing the voltage transformation FFT $(V)$ by the current stimulus transformation FFT $(I)$. The stimulus file was generated by Python-based software, imported into pClamp, and applied as described above. The resonance frequency $\left(f_{\mathrm{R}}\right)$ was determined as the peak of the ZAP profile.

\section{SUPRATHRESHOLD SINUSOIDAL STIMULUS PROTOCOL}

We wanted to introduce a persistent stimulus, which would mimic the pairing of the synaptic potential (EPSP) and the backpropagating spike in the soma, while avoiding actual synaptic plasticity that could complicate the interpretation of the results. We therefore designed a SSS protocol that elicited a single spike each cycle, coinciding with the rising phase of a sinusoidal current 
injected directly to the soma (Figure $\mathbf{3 A}$ ). The stimulus protocol consists of 10 episodes of 10-s long suprathreshold sinusoidal current at $7 \mathrm{~Hz}$ with an inter-episode interval of $5 \mathrm{~s}$, injected through the somatic recording electrode. To determine the stimulus strength, graded sinusoidal currents (10 pA increments) were injected into the soma until a single spike was initiated (sinusoidal rheobase $-\mathrm{Rh}_{\text {sin }}$; see Figure 1). To ensure spike generation in more than $80 \%$ of cycles, the stimulus strength was set to $120-140 \%$ of the $\mathrm{Rh}_{\text {sin }}$, ensuring a single spike at each cycle. The delay of the bAP from the soma to the dendrite was measured from the peak of the intracellularly recorded somatic AP to the peak of the bAP from cell-attached recordings in the dendrites.

\section{STATISTICAL ANALYSIS}

Data is reported as mean \pm SEM. Statistical comparisons were done using two-tailed unpaired Student's $t$-test.

\section{RESULTS}

We have studied the alterations in spike backpropagation delays in layer V pyramidal neurons of the somatosensory cortex. The average somatic resting membrane potential and spike amplitude were $-62 \pm 1$ and $97.5 \pm 4.6 \mathrm{mV}(n=9)$, respectively. Somatic input resistance and time constant were $120 \pm 23 \mathrm{M} \Omega$ and $25 \pm 4 \mathrm{~ms}$ respectively. The average propagation velocity of bAP evoked by step current $(0.2-0.5 \mathrm{nA})$ was $0.68 \pm 0.27 \mathrm{~m} / \mathrm{s}(n=9)$, consistent with previous reports on large layer $\mathrm{V}$ pyramidal neurons in the somatosensory and prefrontal cortex (Stuart et al., 1997a; Gulledge and Stuart, 2003; Bereshpolova et al., 2007). The average resonance frequency in the soma ranges between 1 and $3 \mathrm{~Hz}$ (average $2 \pm 0.2 \mathrm{~Hz} ; n=9$ ) and did not change significantly following the injection of suprathreshold sinusoidal current protocols (Figure 1B).

\section{bAP DELAY IS NOT SUSCEPTIBLE TO TRANSIENT STIMULI}

To assess the bAP timing precision and adaptation to alterations in inputs from the cortical network, we measured the peak-topeak bAP delay via simultaneous recordings from both soma (whole cell) and dendrites (cell attached, up to $500 \mu \mathrm{m}$ from the soma). Previous studies reported on frequency dependence of the spike backpropagation (Spruston et al., 1995; Colbert et al., 1997; Gulledge and Stuart, 2003; Williams, 2004; Williams et al., 2007), in which persistent increase of firing frequencies $(>10 \mathrm{~Hz})$ correlated with higher attenuation of the bAP amplitude. On the other hand, a recent in vivo extracellular study showed that bAP invasion to the dendrites is highly reliable across a wide range of brain states, network activity and stimulus conditions and is only mildly modulated by neuronal firing frequency (Bereshpolova etal., 2007). To examine the dependence of spike backpropagation delay on transient changes in spike firing frequency, we injected a suprathreshold sinusoidal current with increasing frequencies from 0.1 to $100 \mathrm{~Hz}$ (chirp stimulation, Figure 2B) into the soma, which imitates a change in network input, such as the fluctuations in membrane potential ("up" and "down" states), in a more realistic fashion than the step current used previously (Waters and Helmchen, 2004). We then measured the bAP delay time and tested the correlation between the backpropagation delay and the stimulus frequency in which the spikes were initiated.

As seen in Figure 2C, at low frequencies (less than $10 \mathrm{~Hz}$ ), there was a fairly weak correlation between the bAP delay and the

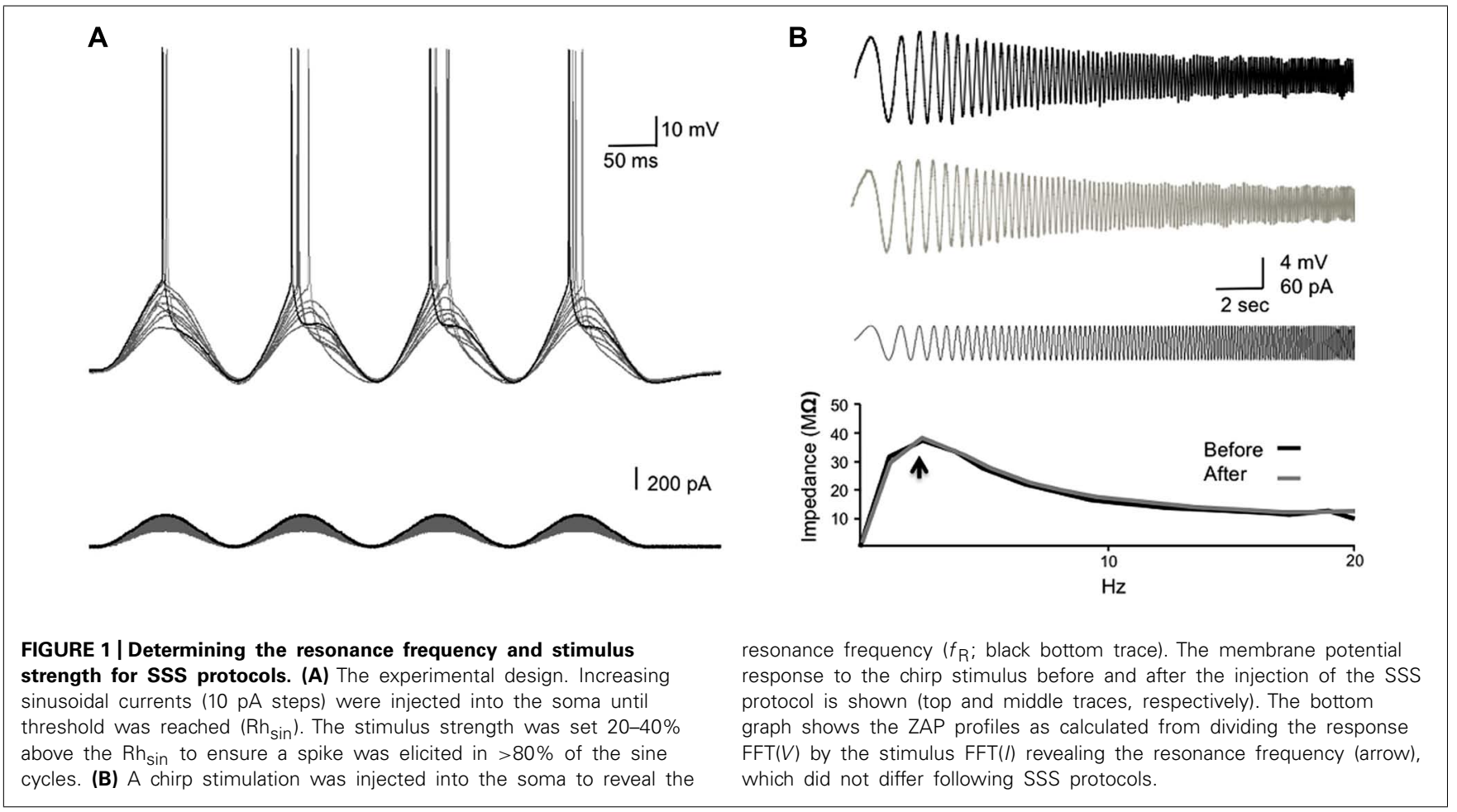




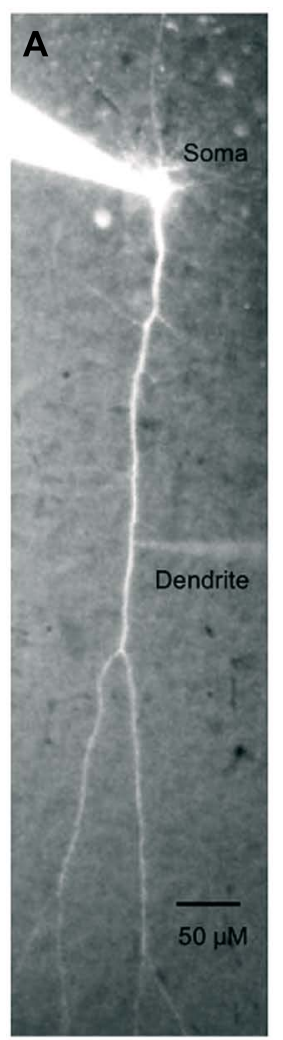

B
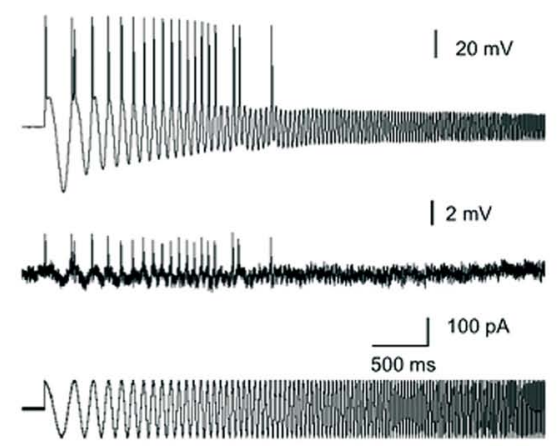

C

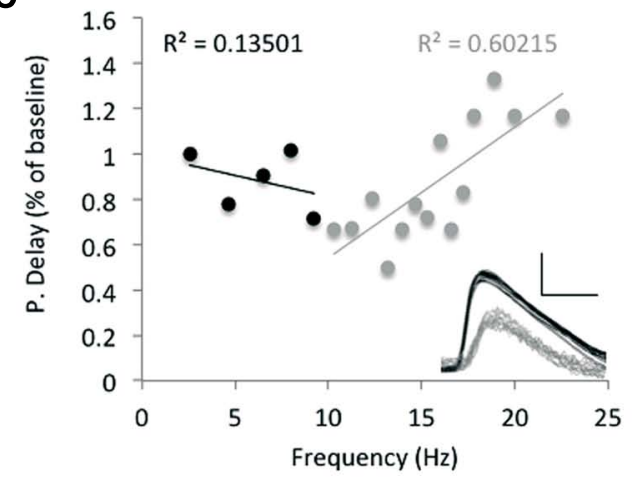

D

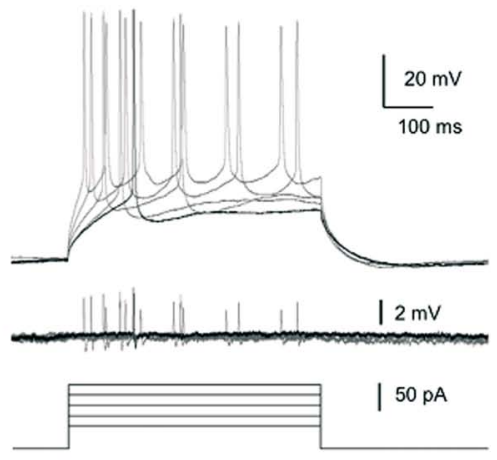

E

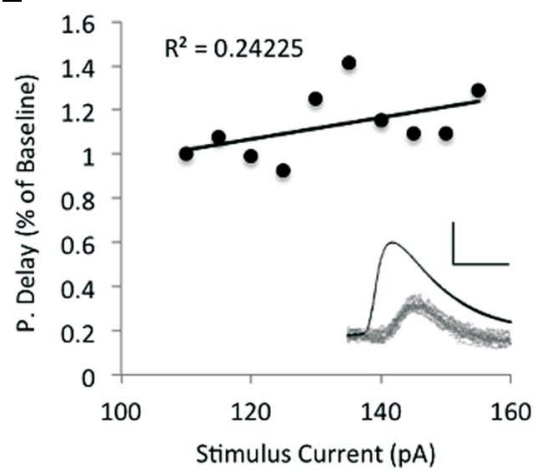

FIGURE 2 |The impact of stimulus intensity and frequency on spike backpropagation delay. (A) Fluorescent image of a layer $V$ pyramidal neuron following intracellular loading with Alexa Fluor-488, showing the cell morphology and placement of the recording electrodes at the soma and dendrite. (B) Propagation delay adaptation following alterations in the stimulus oscillation frequency. Sample traces [200 $\mu \mathrm{m}$ from the soma, baseline propagation velocity $(\mathrm{PV})=0.53 \mathrm{~m} / \mathrm{s}$ ] depict the experimental protocol. A sinusoidal chirp stimulation (bottom trace) was injected into the soma and the spike timing was recorded in both soma and dendrites (top and middle traces, respectively). (C) Scatter plot depicting the correlation between the bAP delay and the stimulus frequency $(n=5)$. Linear correlation was found only at frequencies $>10 \mathrm{~Hz}$ (gray regression line). The propagation delay in the first cycle was used as the baseline.
Inset - sample traces from (B) were superimposed to show the peak-to-peak delay (scale bar - vertical - $40 \mathrm{mV}$ for somatic spikes in black and $2 \mathrm{mV}$ for dendritic spikes in gray; horizontal - $2 \mathrm{~ms}$ ).

(D) Increasing suprathreshold current steps (500 ms, bottom traces) were injected into the soma through the recording electrode and recorded in both soma (top traces) and dendrites (middle traces). The first spike following the stimulus onset was used for comparison in (E). The propagation delay at the lowest stimulus amplitude (110 pA) was used as baseline. (E) Scatter plot depicting the relationship between the injected current intensity and the bAP delay $(n=7)$. Line represents linear fit. Inset - sample traces of the bAP as recorded in the soma and dendrite (350 $\mu \mathrm{m}$ from the soma, baseline PV $=0.46 \mathrm{~m} / \mathrm{s}$ ) are superimposed to show the lack of significant change in each. Scale bar as in (C). stimulus frequency (Linear fit $R^{2}=0.13, n=5$ ), implying that the spike backpropagating delay is not modified by transient changes in the stimulus firing frequency, nor by slow membrane potential oscillations in that range. These results are aligned with a previous in vivo study, which found that firing frequencies below $10 \mathrm{~Hz}$ had only little effect on the extent of backpropagation (Bereshpolova et al., 2007). In contrast, during stimulus frequencies higher than $10 \mathrm{~Hz}$, the bAP delay showed stronger correlation (Linear fit $R^{2}=0.6, n=5$ ) to the stimulus frequency as previously reported for both in vivo and in vitro studies (Spruston et al., 1995; Bereshpolova et al., 2007).

The mechanism underlying STDP involves the coincidence of bAP and EPSP. Previous studies showed that increase in synaptic current reaching the soma could increase the amplitude of bAPs (Sjöström et al., 2001; Stuart, 2001; Stuart and Häusser, 2001) and thereby impact LTP induction. Moreover, it has been shown that increased EPSPs reaching the soma did not affect the action potential amplitude in the soma, but increased its afterdepolarization, which could be associated with bAP amplification. Yet, it is not clear whether modifications of bAP amplitude were due to an increase in the current arriving to the soma per se, or caused by the impact of the increased EPSP along the dendrites and the processes associated with its increase (i.e., calcium influx). The amplitude of EPSP reaching the soma can be mimicked by simple inward current injections into the soma. To assess the impact of the stimulus strength on the spike backpropagation delay, we injected increasing step currents into the soma, which initiated action potentials with decreasing latencies from the stimulus onset as well as steeper increase of the membrane potential rising slopes and increasing afterdepolarizations (Figure 2D), and tested the correlation between the step stimulus strength and the backpropagation delay (Figure 2E). The linear regression line showed only a weak correlation between the stimulus strength and the backpropagation delay $\left(R^{2}=0.24\right)$, which is not 
surprising given the fact that the $\mathrm{Na}^{+}$somatic spike has all or none characteristics.

\section{bAP VELOCITY DECREASES DURING SUPRATHRESHOLD SINUSOIDAL STIMULATION}

The above results indicate that under low frequency stimulation $(<10 \mathrm{~Hz})$, the bAP delay is robust and not affected by transient changes of either stimulus frequency or stimulus amplitude injected to the soma. However, previous studies showed that persistent stimuli, such as an increase in the firing frequency (Spruston et al., 1995; Colbert et al., 1997; Gulledge and Stuart, 2003; Williams, 2004; Williams etal., 2007) or LTP (Dan and Poo, 2004; Sjöström and Häusser, 2006; Williams et al., 2007), that modify the internal cellular environment (i.e., calcium influx and A-type $\mathrm{K}^{+}$inactivation) can result in amplification or attenuation of the bAP amplitude. To study the adaptation of spike backpropagation delay following persistent constant changes in the input stimuli, we injected a suprathreshold sinusoidal current into the soma through the recording electrode, which elicited a single spike each cycle coinciding with the rising phase of the sinusoidal current (Figure 3A). To avoid bAP amplitude attenuation resulting from repetitive high firing rates $(>10 \mathrm{~Hz})$, or bAP amplitude amplifications due to cell resonance, we chose a stimulus frequency that did not result in the attenuation of the bAP amplitude, yet was different from the initial resonance frequency in the soma.

During each episode, the spike initiation phase changed in a constant pattern, starting before the stimulus peak (off-phase) and reaching a steady state after $1 \mathrm{~s}$ (Figures 3B,E). Despite the modifications in the spike initiation phase, the spike shape remained stable in both soma and dendrites during individual episodes (Figure 3D). However, across episodes, a gradual decrease of the average action potential rising slope (10-90\%) was detected in the soma, reaching a significant decrease of $6.3 \pm 0.4 \%$ in the fifth and $8.9 \pm 0.7 \%$ in the last episode $\left(n=6 ; p=1.3 \times 10^{-17}\right.$ and $2 \times 10^{-17}$, respectively, two-tailed Student's $t$-test; Figures 3C and 4C). Although previous studies indicated that the sinusoidal phase of the membrane potential at which the spike was initiated affected the bAP amplitude (Bernard and Johnston, 2003), the sensitivity to small alterations in phase was very minor (compare bAP amplitudes in Figure 2F in Bernard and Johnston, 2003), which is consistent with our results that show no correlation

\section{A}
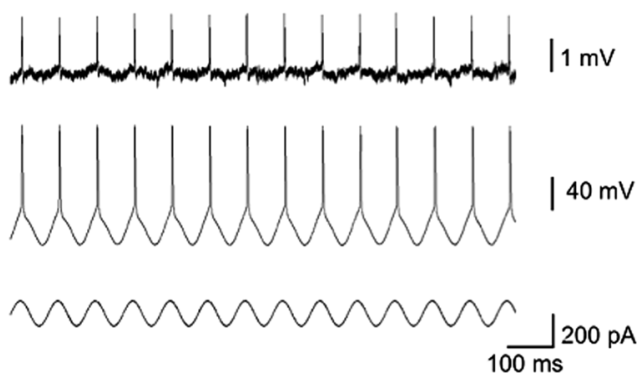

C

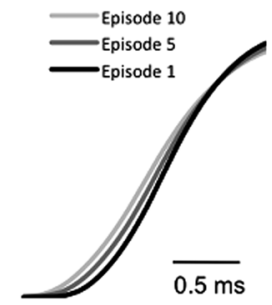

D

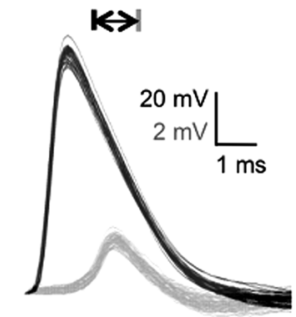

FIGURE 3 | Intrinsic plasticity inducing protocols modify the bAP initiation phase. (A) The experimental design depicting the recording traces from both soma and dendrites during the suprathreshold sinusoidal stimulus protocol, which consists of 10 episodes. In each episode a 10-s suprathreshold sinusoidal current was injected into the soma (bottom trace) at $7 \mathrm{~Hz}$ and elicits spikes in the soma (middle trace), which paired with the peak of each rising cycle and propagated to the dendrites (top trace). (B) A plot depicting the somatic spike-timing phase during SSS protocol. Gray - single episodes, black - average. The horizontal bars represent the first spike in each episode (from left to right, $x$-axis represents episode number), to show the shift of the first spike across

\section{B}

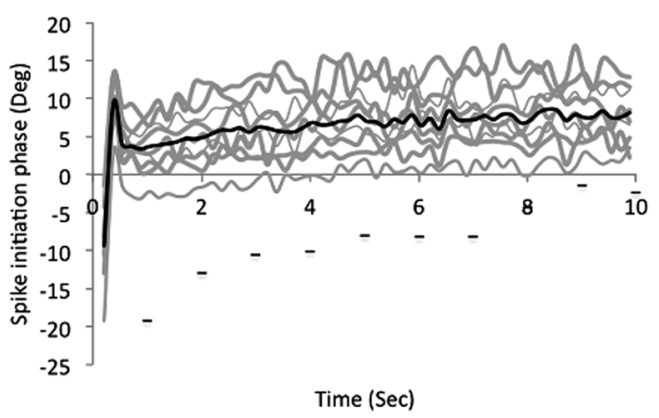

E

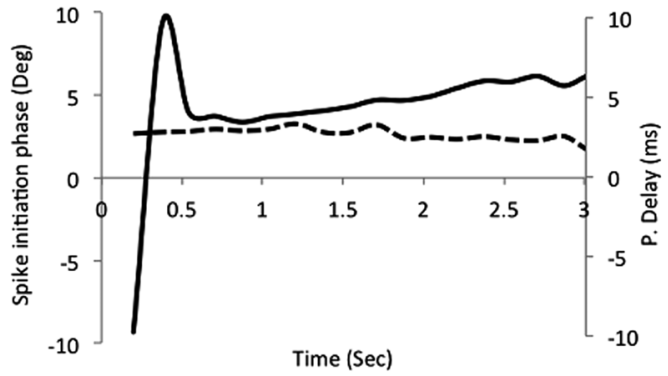

episodes. (C) Expansion of the sample average spike rising slopes of the 1st, 5th, and 10th episodes recorded in the soma depict a decrease of the rising slope across episodes. (D) Expansion of action potentials recorded in the soma (black) and apical dendrite (gray, $350 \mu \mathrm{m}$ from the soma, PV $=0.41 \mathrm{~m} / \mathrm{s}$ ) during one episode. Note the consistent shape and amplitude of spikes during one episode. Scale bar - horizontal $1 \mathrm{~ms}$; vertical $-20 \mathrm{mV}$ black traces and $2 \mathrm{mV}$ gray traces. (E) Expansion of the first $3 \mathrm{~s}$ in (B). Solid line represents the average somatic spike-timing phase. Dashed line represents the average propagation delay time (values on the right ordinate in milliseconds), which did not correlate with the change in the spike initiation phase. 
between the spike initiation phase and the propagation delay (Figure 3E).

The average backpropagation delay gradually increased across sinusoidal stimulus episodes, reaching a significant rise in the third episode. The delay increased by $9 \pm 1 \%$ in the third episode and by $20 \pm 2 \%$ in the 10 th episodes $(n=6 ; p=0.005$ and $1.2 \times 10^{-12}$, respectively, two-tailed Student's $t$-test; Figure 4A), and was accompanied with a significant reduction of the rising slope (10-90\%) of the bAP amplitude in dendrites $(12 \pm 1 \%, n=6$; $p=1.7 \times 10^{-11}$, two-tailed Student's $t$-test; Figures 4B,C) and soma (Figure 4D). The average bAP half-width spike amplitude (HWSA) in the dendrite was $2.39 \pm 0.04 \mathrm{~ms}$ in the first episode and increased significantly (by $12 \pm 1 \%$ ) in the 10 th episode $(p=0.04$, two-tailed Student's $t$-test). As the immediate effect of bAP is the increase in calcium concentration in the dendrites, and previous studies indicated that the intrinsic excitability in the dendrites is calcium dependent (Berridge, 1998), we tested the impact of calcium decrease on bAP during SSS protocol. The addition of the calcium chelator BAPTA $(10 \mathrm{mM})$ into the recording electrode abolished the significant increase of the bAP delay across episodes ( $n=4, p=0.55$, two-tailed Student's $t$-test, comparing the 1 st and 10th episode; Figure 4A), implying a calcium-dependent process in the change of bAP delay. As seen in Figures 4E,F, during the application of the SSS protocol in the presence of BAPTA, the average somatic HWSA increased by $11.7 \pm 0.2 \%(p<0.05$, twotailed $t$-test) and was also accompanied with a significant decrease of the rising slope $(10-90 \%)$ by $17.3 \pm 0.3 \%$ ( $p<0.05$, two-tailed $t$-test). However, calcium chelation by BAPTA had a profound impact on both HWSA and rising slope of the dendritic bAP, eliminating the significant modifications across episodes (compare Figures 4C,F).

\section{DISCUSSION}

We have studied the adaptation of spike backpropagation delays in layer $\mathrm{V}$ pyramidal neurons of the somatosensory cortex during membrane oscillatory regimes.

Under our experimental conditions, the average propagation delay across episodes of SSS protocols increased in an accumulative fashion, until it reached steady state in the fifth episode (>300 spikes; Figure 4A). The phase of spike initiation within each episode changed in a unique pattern which was similar to the somatic action potential repolarization curve following a train of action potentials, described previously by Fleidervish et al. (1996). Thus we assume that the modifications in the spike initiation

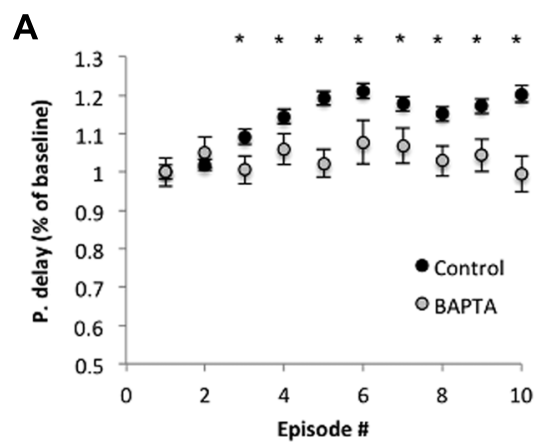

D

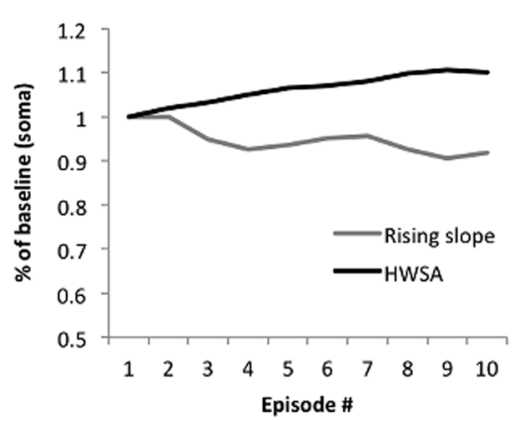

B

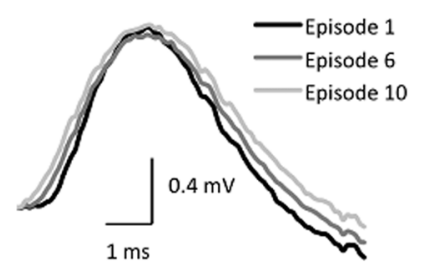

$\mathbf{E}$

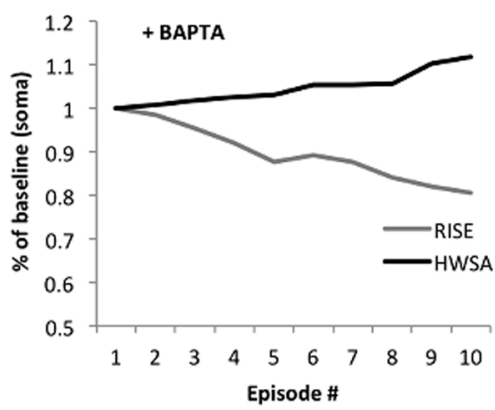

C

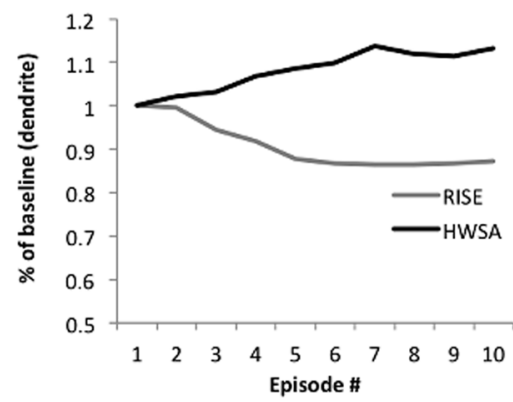

$\mathbf{F}$

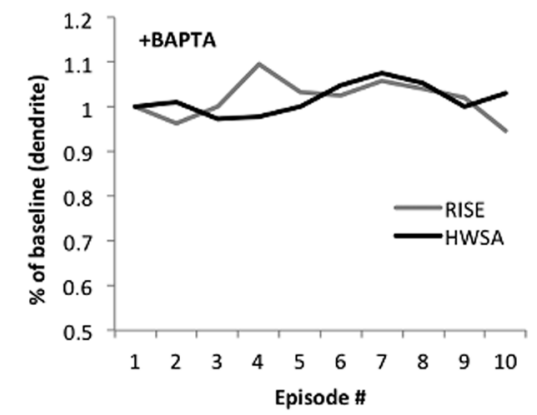

FIGURE 4 | Suprathreshold sinusoidal stimulus protocols modify spike backpropagation delays. (A) Average propagation delay (shown as \% of baseline) during the SSS protocol. Each dot represents the average delay in the episode. The average delay in the first episode was used as baseline. Significant alterations from baseline were noticed from the third episode onward ( ${ }^{*} p<0.005$, two-tailed Student's $t$-test). The calcium chelator BAPTA abolished the significant increase in propagation delay in the last seven episodes, implying a calcium dependence $(n=4, p>0.3$; two-tailed Student's $t$-test). (B) Average bAP traces in the dendrite $(250 \mu \mathrm{m}$ from the soma, aligned to the peak) at the $1 \mathrm{st}, 6 \mathrm{th}$, and 10th episode show the changes in the slope. Scale bar (1 ms; $0.4 \mathrm{mV})$. (C,F) The average rising slope $(10-90 \%)$ and HWSA of the bAP recorded in the dendrite during SSS show decreases and increases, respectively, across episodes as a \% of baseline, which determined as the average rising slope in the first episode.

(D,E) Modifications of the spike waveform across episodes plotted as a change from baseline. Black - HWSA, gray - 10-90\% rising slope. (F) The addition of BAPTA to the recording electrode modified the significant alterations in the spike waveform. 
phase within each episode (Figure 3B) resembles spike frequency adaptation, which may be attributed to the fast inactivation of the $\mathrm{Na}^{+}$current and positive shift of the threshold potential. We also observed a gradual modification of the first spike initiation phase of each episode within the SSS protocol (bars in Figure 3B), which might be attributed to the slow recovery of the $\mathrm{Na}^{+}$channels from inactivation as reported previously (Fleidervish et al., 1996; Colbert et al., 1997). Yet, the alterations in the spike initiation phase during the episode did not affect the backpropagation velocity (Figure 2E). Moreover, at low frequencies $(<10 \mathrm{~Hz})$, transient alterations in the stimulus oscillation as well as modifications of the stimulus strength (Figure 2) showed only weak correlation to the backpropagation delay, implying that the bAP delay has robust features, which are not easily modified. These findings are aligned with previous in vivo study showing that at low frequencies the degree of bAP under varying conditions is quite uniform (Bereshpolova et al., 2007). However, during higher suprathreshold stimulus frequencies $(10-25 \mathrm{~Hz})$, the relationship between stimulus frequency and bAP delays showed higher correlation, which could be attributed to a calcium "dendritic switch" (reviewed by Sjöström et al., 2008), in which calcium influx into the apical dendrite is stronger with increasing network activity, hence boosting spike backpropagation. This further suggests that backpropagation can be modulated by the network state to play a role in cortical plasticity (Waters and Helmchen, 2004; Bereshpolova et al., 2007; Sjöström et al., 2008).

Alterations in the bAP delay could potentially be measured using two components. The first component can be detected through changes in the bAP waveform (i.e., decrease in the rising slope as seen in Figures 4B,C and widening of the HWSA), which are mainly due to modifications of the voltage-gated sodium current and $\mathrm{Ca}^{2+}$-dependent $\mathrm{K}^{+}$channels. The second component is the propagation time along the dendrite, which can be measured by subtracting the changes in bAP rise time recorded in the dendrite from the total propagation and compare it to the original propagation delay. During the SSS protocol, the average rising slope of the bAP in the dendrite at the tenth episode decreases by $12 \pm 1 \%$, while the total increase in the propagation delay was $20 \pm 2 \%$. These results signify that under our experimental design, the alterations in the propagation delay include both components.

Migliore (1996) suggested two possibilities for spike attenuation and active propagation in the dendrites (see also Magee et al., 1998). The first is slow inactivation of the $\mathrm{Na}^{+}$channels (due to the repetitive firing) which was experimentally reported (Colbert et al., 1997; Jung et al., 1997; Colbert and Johnston, 1998), and the other is through activation and inactivation of the calciumdependent $\mathrm{K}^{+}$channels (Jaffe et al., 1992; Bekkers, 2000), also called shunting conductance. Other factors that might impact the bAP propagation involves the increase in calcium concentration along the dendrite, which can boost the amplification of depolarizing events as well as voltage-dependent currents (such as $I_{\mathrm{h}}$ ) that are activated during the membrane oscillation.

\section{bAP DELAY IS AFFECTED BY CALCIUM-DEPENDENT PROCESSES}

Various modulators may regulate spike backpropagation (Colbert et al., 1997; Astman et al., 1998; Hoffman and Johnston,
1998; Sjöström etal., 2008), yet, the principal factor governing spike initiation and propagation is the ratio between the $\mathrm{Na}^{+} / \mathrm{K}^{+}$currents available at each location. The immediate effect of bAP is the increase in calcium entry into the dendrites through voltage-gated $\mathrm{Ca}^{2+}$ channels, which then affects $\mathrm{Ca}^{2+}$ dependent downstream processes such as $\mathrm{Ca}^{2+}$-activated $\mathrm{K}^{+}$ conductance as well as slow inactivation of the $\mathrm{Na}^{+}$conductance (Jaffe et al., 1992; Markram et al., 1997; Tsubokawa and Ross, 1997). That the gradual enhancement in propagation delay across episodes of the SSS protocol was due to a calcium-dependent process was shown by its ablation by the addition of the calcium chelator BAPTA to the recording electrode in the soma (Figure 4A). As calcium is involved in numerous processes that can influence dendritic excitability over a time period of minutes (Berridge, 1998), and it affects both rising slope and HWSA of the bAP in the dendrite (Figure 4F) we assume that multiple processes and not a single mechanism disrupt the adaptation of the bAP delay under BAPTA. These results are also aligned with a previous study by (Su et al., 2001), which suggests that a decrease in calcium concentration enhances intrinsic bursting via an increase of the persistent $\mathrm{Na}^{+}$current. Hence, a reduction in calcium concentration upmodulates the persistent $\mathrm{Na}^{+}$current by shifting its activation threshold (Li and Hatton, 1996), thus enhancing propagation velocity and reducing the delay.

The time course of the delay alterations were in the order of tens of seconds to minutes (Figures 4A,B), implying that the mechanism underlying this modification is probably mediated through a second messenger system, such as modulation of $\mathrm{K}^{+}$channels by protein kinase $\mathrm{A}$ (PKA), or inactivation of $\mathrm{Na}^{+}$channels by the $\mathrm{Ca}^{2+}$-dependent protein kinase $\mathrm{C}(\mathrm{PKC})$, as suggested by Hoffman and Johnston (1998). Activation of PKC has a dual impact on $\mathrm{Na}^{+}$currents. On one hand it modulates the inactivation of $\mathrm{Na}^{+}$channels, thus decreases their immediate availability, while on the other hand, on time scale of minutes, it shifts their voltage-dependent activation curve toward a more hyperpolarized potential (Astman et al., 1998), thus reducing spike threshold and changing both spike backpropagation amplitude and delay (Colbert and Johnston, 1998).

We therefore propose that following persistent repetitive invasion of bAPs to the dendrites, there is an increase in calcium influx, activation of $\mathrm{Ca}^{2+}$-dependent $\mathrm{K}^{+}$currents (Bekkers, 2000) and fast inactivation of $\mathrm{Na}^{+}$channels. These processes have fast kinetics, which alter the spike initiation phase in each episode as seen in Figure 3D. At later stages, the calcium influx leads to activation of PKC- and cAMP-dependent pathways as suggested previously (Astman et al., 1998; Colbert and Johnston, 1998; Hoffman and Johnston, 1998). These modifications further result in phosphorylation (and slow inactivation) of voltagegated $\mathrm{Na}^{+}$channels in both soma and dendrites. As dendritic and somatic $\mathrm{Na}^{+}$currents recover with different time constants (5.6 and 4.1 s, respectively; see Colbert et al., 1997), the discrepancy between the dendritic recovery time to the somatic one shifts the $\mathrm{Na}^{+} / \mathrm{K}^{+}$conductance ratio, which could explain the delay adaptation we are seeing across episodes. As the bAP is a regenerative process, a change in its voltage threshold due 
to different ratios of $\mathrm{Na}^{+} / \mathrm{K}^{+}$conductance's will result in a change of the propagation delay. Further investigation into the kinetics and range of the backpropagation delay adaptation following SSS will enhance our understanding of the underling processes.

\section{REFERENCES}

Astman, N., Gutnick, M. J., and Fleidervish, I. A. (1998). Activation of protein kinase $\mathrm{C}$ increases neuronal excitability by regulating persistent $\mathrm{Na}^{+}$current in mouse neocortical slices. J. Neurophysiol. 80, 1547-1551.

Bekkers, J. M. (2000). Distribution and activation of voltage-gated potassium channels in cell-attached and outside-out patches from large layer 5 cortical pyramidal neurons of the rat. J. Physiol. 525, 611-620. doi: 10.1111/j.1469-7793.2000.t01-200611.x

Bereshpolova, Y., Amitai, Y., Gusev, A. G., Stoelzel, C. R., and Swadlow, H. A. (2007). Dendritic backpropagation and the state of the awake neocortex. J. Neurosci. 27, 9392-9399. doi: 10.1523/JNEUROSCI.2218-07.2007

Bernard, C., and Johnston, D. (2003). Distance-dependent modifiable threshold for action potential back-propagation in hippocampal dendrites. J. Neurophysiol. 90, 18071816. doi: 10.1152/jn.00286.2003

Berridge, M. J. (1998). Neuronal calcium signaling. Neuron $21,13-$ 26. doi: 10.1016/S0896-6273(00) 80510-3

Bienenstock, E. L., Cooper, L. N., and Munro, P. W. (1982). Theory for the development of neuron selectivity: orientation specificity and binocular interaction in visual cortex. $J$. Neurosci. 2, 32-48.

Buskila, Y., and Amitai, Y. (2010). Astrocytic iNOS-dependent enhancement of synaptic release in mouse neocortex. J. Neurophysiol. 103, 1322-1328. doi: 10.1152/jn.00676.2009

Buskila, Y., Wright, J., and Vanschaik, A. (2013). "Membrane oscillations plasticity and signal propagation delays in spiking neurons," in Poster at the Australian Neuroscience Society Annual Meeting, POS-MON038, Melbourne, February 3-6, 2013.

Colbert, C., and Johnston, D. (1998). Protein kinase $\mathrm{C}$ activation decreases activity-dependent attenuation of dendritic $\mathrm{Na}^{+}$current in hippocampal CA1 pyramidal neurons. J. Neurophysiol. 79, 491-495.

Colbert, C. M., Magee, J. C., Hoffman, D. A., and Johnston, D. (1997). Slow recovery from inactivation of $\mathrm{Na}^{+}$channels underlies the activitydependent attenuation of dendritic action potentials in hippocampal
CA1 pyramidal neurons. J. Neurosci. 17, 6512-6521.

Dan, Y., and Poo, M. (2006). Spike timing-dependent plasticity: from synapse to perception. Physiol. Rev. 86, 1033-1048. doi: 10.1152/physrev.00030.2005

Dan, Y., and Poo, M.-M. (2004). Spike timing-dependent plasticity of neural circuits. Neuron 44, 23-30. doi: 10.1016/j.neuron.2004.09.007

Fleidervish, I. A., Friedman, A., and Gutnick, M. J. (1996). Slow inactivation of $\mathrm{Na}^{+}$current and slow cumulative spike adaptation in mouse and guinea-pig neocortical neurones in slices. J. Physiol. 493(Pt 1), 83-97.

Froemke, R. C., Letzkus, J. J., Kampa B. M., Hang, G. B., and Stuart, G. J. (2010). Dendritic synapse location and neocortical spike-timingdependent plasticity. Front. Synaptic Neurosci. 2:29. doi: 10.3389/fnsyn. 2010.00029

Gulledge, A. T., and Stuart, G. J. (2003) Action potential initiation and propagation in layer 5 pyramidal neurons of the rat prefrontal cortex: absence of dopamine modulation. J. Neurosci. 23, 11363-11372.

Gutfreund, Y., Yarom, Y., and Segev, I. (1995). Subthreshold oscillations and resonant frequency in guineapig cortical neurons: physiology and modelling. J. Physiol. 483, 621-640.

Häusser, M., and Mel, B. (2003). Dendrites: bug or feature? Curr. Opin. Neurobiol. 13, 372-383. doi: 10.1016/S0959-4388(03)00075-8

Hoffman, D. A., and Johnston, D. (1998). Downregulation of transient $\mathrm{K}^{+}$channels in dendrites of hippocampal CA1 pyramidal neurons by activation of PKA and PKC. J. Neurosci. 18, 3521-3528.

Jaffe, D., Johnston, D., and Lasser-Ross N. (1992). The spread of $\mathrm{Na}^{+}$spikes determines the pattern of $\mathrm{Ca}^{2+}$ entry into hippocampal neurons. Nature 357, 244-246. doi: 10.1038/357244a0

Johnston, D., and Hoffman, D. (1999). Regulation of back-propagating action potentials in hippocampal neurons. Curr. Opin. Neurobiol. 9, 288-292. doi: 10.1016/S0959-4388 (99) $80042-7$

Jung, H. Y., Mickus, T., and Spruston, N. (1997). Prolonged sodium channel inactivation contributes to dendritic action potential

\section{AUTHOR CONTRIBUTIONS}

Yossi Buskila, John W. Morley, Jonathan Tapson, and André van Schaik designed the approach; Yossi Buskila performed the measurements and analyzed the electrophysiological recordings. All authors were involved in writing the paper.

attenuation in hippocampal pyramidal neurons. J. Neurosci. 17, 66396646.

Larkum, M. (1999). Calcium electrogenesis in distal apical dendrites of layer 5 pyramidal cells at a critical frequency of action potentials. Proc. Natl. Acad. Sci. U.S.A. 96, 14600-14604. doi: 10.1073/pnas. 96.25 .14600

Li, Z., and Hatton, G. I. (1996). Oscillatory bursting of phasically firing rat supraoptic neurones in low- $\mathrm{Ca}^{2+}$ medium: $\mathrm{Na}^{+}$influx, cytosolic $\mathrm{Ca}^{2+}$ and gap junctions. J. Physiol. 496(Pt 2), 379-394

Magee, J., Hoffman, D., Colbert, C., and Johnston, D. (1998). Electrical and calcium signaling in dendrites of hippocampal pyramidal neurons. Annu. Rev. Physiol. 60, 327-346. doi: 10.1146/annurev.physiol.60.1. 327

Magee, J. C., and Johnston, D. (1997) A synaptically controlled, associative signal for Hebbian plasticity in hippocampal neurons. Science 275, 209-213. doi: 10.1126/science.275.5297.209

Markram, H., Lübke, J., Frotscher, M., and Sakmann, B. (1997). Regulation of synaptic efficacy by coincidence of postsynaptic APs and EPSPs. Sci ence $275,213-215$. doi: $10.1126 / \mathrm{sci}$ ence. 275.5297 .213

Migliore, M. (1996). Modeling the attenuation and failure of action potentials in the dendrites of hippocampal neurons. Biophys. J. 71, 2394-2403. doi:10.1016/S00063495(96)79433-X

Narayanan, R., and Johnston, D. (2010). The $h$ current is a candidate mechanism for regulating the sliding modification threshold in a BCM-like synaptic learning rule. J. Neurophysiol. 104, 1020-1033. doi: 10.1152/jn.01129.2009

Nevian, T., Larkum, M. E., Polsky, A., and Schiller, J. (2007). Properties of basal dendrites of layer 5 pyramidal neurons: a direct patch-clamp recording study. Nat. Neurosci. 10, 206-214. doi: 10.1038/nn1826

Sjöström, P., Rancz, E., Roth, A. and Häusser, M. (2008). Dendritic excitability and synaptic plasticity. Physiol. Rev. 88, 769-840. doi: 10.1152/physrev.00016.2007

Sjöström, P. J., and Häusser, M. (2006). A cooperative switch determines the sign of synaptic plasticity in distal dendrites of neocortical pyramidal neurons. Neuron 51, 227238. doi: 10.1016/j.neuron.2006.06. 017

Sjöström, P. J., Turrigiano, G. G., and Nelson, S. B. (2001). Rate, timing, and cooperativity jointly determine cortical synaptic plasticity. Neuron 32, 1149-1164. doi: $\quad 10.1016 / S 0896-6273(01) 005$ 42-6

Spruston, N., Schiller, Y., Stuart, G., and Sakmann, B. (1995). Activitydependent action potential invasion and calcium influx into hippocampal CAl dendrites. Science 268, 297-300. doi: 10.1126/science. 7716524

Stuart, G., and Sakmann, B. (1995). Amplification of EPSPs by axosomatic sodium channels in neocortical pyramidal neurons. Neuron 15, 1065-1076. doi: 10.1016/08966273(95)90095-0

Stuart, G., Schiller, J., and Sakmann, B. (1997a). Action potential initiation and propagation in rat neocortical pyramidal neurons. J. Physiol. 505(Pt 3), 617-632

Stuart, G., Spruston, N., Sakmann, B., and Häusser, M. (1997b). Action potential initiation and backpropagation in neurons of the mammalian CNS. Trends Neurosci. 20, 125-131.

Stuart, G. J. (2001). Determinants of spike timing-dependent synaptic plasticity. Neuron 32, 966-968. doi: 10.1016/S0896-6273(01)00541-4

Stuart, G. J., and Häusser, M. (2001). Dendritic coincidence detection of EPSPs and action potentials. Nat. Neurosci. 4, 63-71. doi: $10.1038 / 82910$

Su, H., Alroy, G., Kirson, E. D., and Yaari, Y. (2001). Extracellular calcium modulates persistent sodium current-dependent burst-firing in hippocampal pyramidal neurons. J. Neurosci. 21, 4173-4182.

Tsubokawa, H., and Ross, W. (1997). Muscarinic modulation of spike backpropagation in the apical dendrites of hippocampal CA1 pyramidal neurons. J. Neurosci. 17, 5782-5791.

Vetter, P., Roth, A., and Häusser, M. (2001). Propagation of action potentials in dendrites depends on dendritic morphology. J. Neurophysiol. 85, 926-937. 
Waters, J., and Helmchen, F. (2004). Boosting of action potential backpropagation by neocortical network activity in vivo. $J$. Neurosci. 24, 11127-11136. doi: 10.1523/JNEUROSCI.2933-04.2004

Waters, J., Schaefer, A., and Sakmann, B. (2005). Backpropagating action potentials in neurones: measurement, mechanisms and potential functions. Prog. Biophys. Mol. Biol. 87, 145-70. doi: 10.1016/j.pbiomolbio.2004.06. 009
Williams, S. R. (2004). Spatial compartmentalization and functional impact of conductance in pyramidal neurons. Nat. Neurosci. 7, 961-967. doi: 10.1038/nn1305

Williams, S. R., Wozny, C., and Mitchell, S. J. (2007). The back and forth of dendritic plasticity. Neuron 56, 947-953. doi: 10.1016/j.neuron.2007.12.004

Conflict of Interest Statement: The authors declare that the research was conducted in the absence of any commercial or financial relationships that could be construed as a potential conflict of interest.

Received: 13 July 2013; accepted: 07 October 2013; published online: 30 October 2013.

Citation: Buskila Y, Morley JW, Tapson $J$ and van Schaik A (2013) The adaptation of spike backpropagation delays in cortical neurons. Front. Cell. Neurosci. 7:192. doi: 10.3389/fncel.2013. 00192
This article was submitted to the journal Frontiers in Cellular Neuroscience

Copyright (c) 2013 Buskila, Morley, Tapson and van Schaik. This is an open-access article distributed under the terms of the Creative Commons Attribution License (CC BY). The use, distribution or reproduction in other forums is permitted, provided the original author(s) or licensor are credited and that the original publication in this journal is cited, in accordance with accepted academic practice. No use, distribution or reproduction is permitted which does not comply with these terms. 